\title{
The ISNaS Compressible Navier-Stokes Solver; First Results for Single Airfoils ${ }^{1}$
}

\author{
F.J. Brandsma ${ }^{1}$, J.G.M. Kuerten ${ }^{2}$ \\ ${ }^{1}$ National Aerospace Laboratory, Aerodynamics Division, P.O. Box 90502, \\ 1006 BM Amsterdam, The Netherlands \\ ${ }^{2}$ Department of Applied Mathematics, University of Twente, P.O. Box 217, \\ 7500 AE Enschede, The Netherlands
}

\section{Introduction}

In the ISNaS project, an information system for flow simulation based on the NavierStokes equations is being developed. As part of this project the National Aerospace Laboratory and the University of Twente cooperate on the development of a flow solver for three-dimensional, compressible flow based on the Navier-Stokes equations. The first application will be multi-element airfoils with extended flaps and slats for take-off and landing conditions, taking into account infinite-sheared wing effects. Because of the complex geometrical shape of this application, it has been decided to develop a multi-block solver. The computational domain is divided into blocks, where in each block a boundaryconforming, structured grid is used. This choice facilitates the easy use of the Euler equations in the outer regions of the flow, where viscous effects are negligible, whereas the more expensive evaluation of the Navier-Stokes equations can be restricted to the viscous, inner regions of the flow (boundary layers and wakes). The flow solver will be based on the Reynolds-averaged Navier-Stokes equations, where initially the Baldwin-Lomax turbulence model will be used [1].

A stepwise development of the flow solver has been planned, starting from inviscid flow, via viscous, laminar flow to viscous, turbulent flow, each around a single airfoil. Subsequently, the integration scheme will be implemented in a multi-block solver. After each development step the flow solver will be validated in view of requirements with respect to numerics, performance and flow physics. In this paper results of the monoblock solver for viscous, laminar flow over a flat plate, and viscous, turbulent flow around a single airfoil will be presented. Implementation of the multi-block solver is in progress.

1 The ISNaS project is partly subsidized by the Dutch Ministries of Education and Sciences, and of Transport and Public Works. Part of the necessary supercomputer time is subsidized by 'Stichting SURF' from the 'Nationaal Fonds gebruik Supercomputers (NFS)' 


\section{Numerical Method}

The unsteady, three-dimensional, Reynolds-averaged Navier-Stokes equations for compressible flow are integrated in time towards a steady state solution. The equations are written in integral form, where density, total energy density and the three Cartesian components of the momentum density are used as dependent variables.

Since the ISNaS project aims at applications on the industrial level, the numerical method must be robust and has therefore been selected from the group of well-proven integration schemes. This has led to a time-explicit scheme, using a finite-volume discretization in physical space. In order to cope with the highly non-uniform grids which are typical for the applications of interest, the present solver employs a cell-vertex technique instead of the commonly used cell-centred approach. For each vertex the control volume is the union of the eight grid cells meeting at that vertex.

For the spatial discretization central differences are used combined with second- and fourth order artificial dissipation. The fourth order dissipation is necessary to prevent odd-even decoupling, whereas the second order dissipation makes it possible to capture shocks. This formulation is based on the work of Jameson [2] for simulation of inviscid flow. For viscous flow several modifications are necessary in order to minimize the numerical dissipation in boundary layers and wakes [3]. As boundary conditions at the solid surface the velocity components are set equal to zero, while the density and energy density at the surface are found by solving the continuity and energy equations, assuming an adiabatic wall. The treatment of the far-field boundaries is based on approximate Riemann invariants, where in case of two-dimensional applications a circulation correction (bound vortex formulation) is added to the free stream quantities [4].

Integration in time is performed using a multistage Runge-Kutta scheme. Since the objective is the calculation of the steady state solution (no time accuracy is pursued), the coefficients in the Runge-Kutta scheme are chosen in such a way that the stability region is as large as possible. For the same reason local timestepping is applied to accelerate the convergence. For the time being no other convergence acceleration techniques, like residual averaging and multigrid, are considered, in order to avoid difficulties at block interfaces. At present, both a three-stage and a five-stage scheme are used. In the threestage scheme the dissipative terms are evaluated only at the first stage, while in the five-stage scheme they are recalculated at the third and at the fifth stage.

\section{Results}

\subsection{Laminar Flow over a Flat Plate}

The flow solver has been validated for laminar flow by comparing the results for flow over a flat plate with analytical solutions. To this end the two-dimensional subsonic laminar non-lifting flow over a finite flat plate at free-stream Mach number $M_{\infty}=0.3$, and Reynolds number $R e_{\infty}=10^{4}$ (based on the platelength), is considered. A rectangular grid with 312 cells in streamwise direction (256 on the plate) and 128 in normal direction is used. The grid points in streamwise direction are clustered around the leading and trailing edge with the smallest distance $5 \times 10^{-4}$, in order to resolve the local small scale phenomena. The grid spacing in normal direction is stretched, with the first grid 
line at a distance of $1.8 \times 10^{-4}$ platelengths from the wall. The computational domain extends from 0.5 platelengths upstream till 25 platelengths downstream of the plate, and 1 platelength above the plate. For the low Mach number used here, the numerical solution should compare well with the analytical solutions for incompressible flow. Above the plate, away from the leading and trailing edge, the analytical solution is determined by the classical Blasius theory. Figure 1 shows that the agreement between the calculated velocity profiles and the Blasius solution is good.

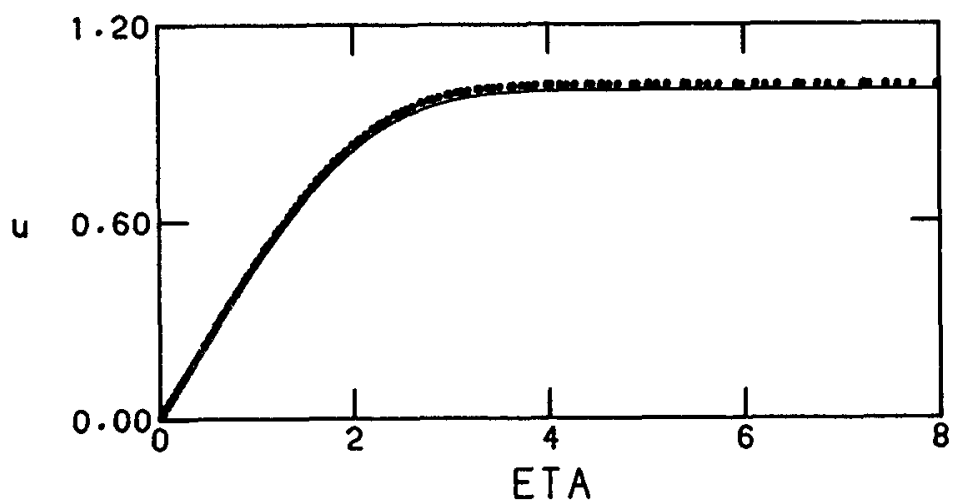

Fig. 1. Comparison of the calculated velocity profiles (nondimensional velocity $u$ against scaled normal distance $\mathrm{ETA}=z \sqrt{R e_{\infty} / 2 x}$ ) between 0.4 and 0.6 platelengths with the Blasius solution (solid line)

In figure 2 the calculated velocity at the wake centre-line is compared with the analytical solution, deduced from asymptotic theory in several flow regimes. Theoretically three regions can be distinguished here: immediately behind the trailing edge there is a small region of $\mathcal{O}\left(R e_{\infty}^{-3 / 4}\right)$ platelengths where the full Navier-Stokes equations should be considered [5]; at larger distances from the trailing edge the flow is well described by the triple-deck approximation [6]; this triple-deck merges with the classical Goldstein solution, which is valid up to downstream infinity. As can be seen, in all three regimes the comparison between calculation and theory is good. This is clearly demonstrated by the asymptotic behaviour for vanishing distance $x$ from the trailing edge. Theoretically, for $R e_{\infty}=10^{4}$ the wake centre-line velocity behaves as $1.39 \sqrt{ } x$ near the trailing edge, whereas the calculation yields $u \sim 1.37 \sqrt{ } x$. The calculated drag coefficient $\left(C_{D}=0.02822\right)$ compares excellently with the analytical triple-deck value (0.02823) [6].

\subsection{Turbulent Flow around an Airfoil}

A good testcase for turbulent flow over a lifting airfoil is RAE2822 case $6: M_{\infty}=$ $0.725, R e_{\infty}=6.5 \times 10^{6}, \alpha=2.92^{\circ}$ (uncorr.). For this testcase well-documented experimental results are available [7], and this case was also included in the 'Holst workshop' [8]. The calculations have been performed on a C-type grid with 352 cells in streamwise direction ( 256 cells around the airfoil) and 64 cells in normal direction. The first grid line 
u

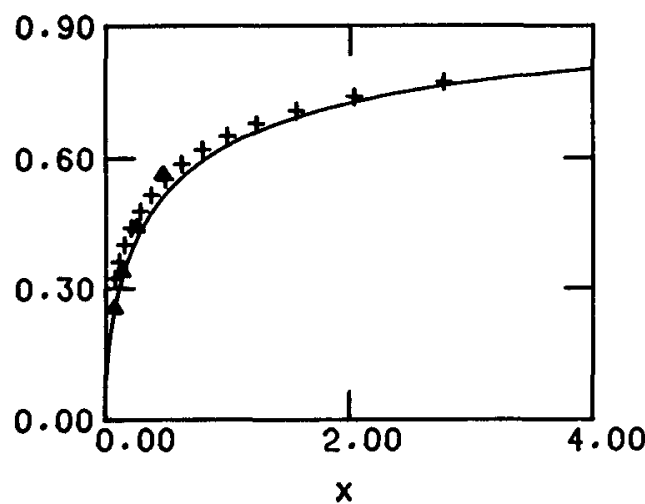

u

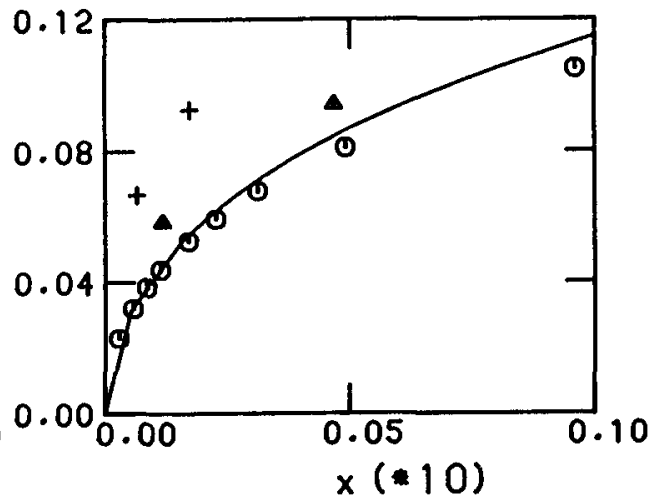

Fig. 2. Comparison of the velocity at the wake centre line (nondimensional velocity $u$ against nondimensional distance from the trailing edge $x$ ) between calculations with the ISNaS solver (solid line) and asymptotic theory (o: Navier-Stokes; $\Delta$ : triple-deck; +: Goldstein)

above the airfoil is chosen in such a way that the law-of-the-wall coordinate $y^{+}$is close to 1 along this line. The far-field boundary is located at a distance of approximately 10 chordlenghts away from the airfoil. For the calculations the angle of incidence was chosen to be $\alpha=2.44^{\circ}$ according to the windtunnel corrections indicated in [7]. A total number of 12,000 iterations has been performed, resulting in a reduction of more than four orders of magnitude of the density residuals.

In figure 3 the results of the ISNaS flow solver for this case are compared with experimental data. For the pressure distribution the overall agreement is good. The small discrepancies on the lower surface, and on the upper surface just behind the suction peak and near the shock, are comparable with most of the other numerical results for this case presented in the Holst workshop [8]. The calculated lift coefficient $\left(C_{L}=0.772\right)$ is larger than the experimental value (0.743). The boundary layer on the upper surface is well resolved, as may be concluded from the agreement between the experimental and calculated displacement thickness. The calculated drag coefficient $\left(C_{D}=0.0125\right)$ is close to the experimental value $(0.0127)$.

\section{Conclusions}

This paper describes the cell-vertex scheme for the compressible Navier-Stokes equations, to be part of the ISNaS flow simulation system. A comparison between numerical and analytical results for viscous, laminar flow shows that boundary layers and wakes are correctly resolved. Also for transonic turbulent flow accurate results have been obtained, as can be seen from a comparison with experimental results and other numerical solutions for the RAE2822 testcase. 
CP

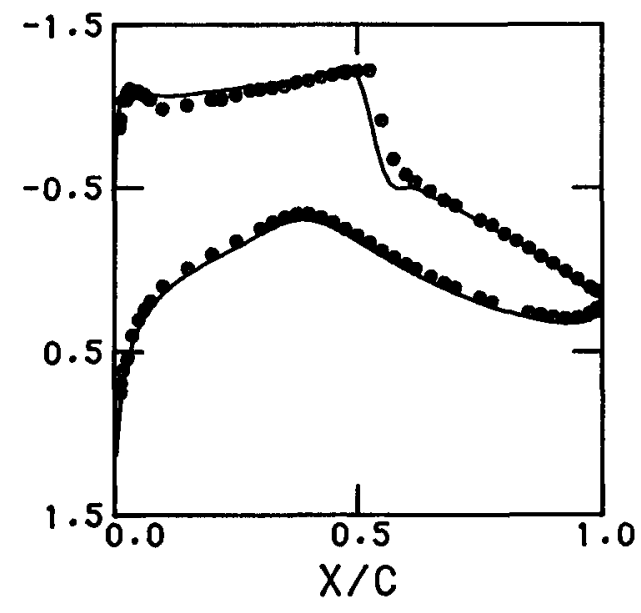

$8 \%(-10)$

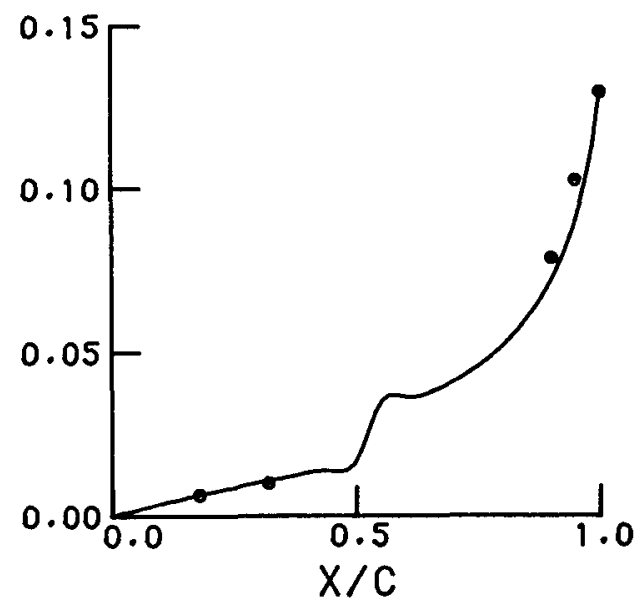

Fig. 3. RAE2822 case $6\left(M_{\infty}=0.725, R e_{\infty}=6.5 \times 10^{6}, \alpha=2.92^{\circ}\right)$ : comparison of the ISNaS results with experimental data. left: pressure distribution; right: displacement thickness on the upper surface; o: experiment; solid line: calculation

\section{References}

1. F.J. Brandsma: Mathematical Physics Aspects of Simulations based on the Navier-Stokes Equations, ISNaS 88.12.024 / NLR TP 89069 L (1989)

2. A. Jameson, W. Schmidt, E. Turkel: Numerical Solutions of the Euler Equations by Finite Volume Methods Using Runge-Kutta Time-Stepping-Schemes, AIAA Paper 81-1259 (1981)

3. J.G.M. Kuerten: Numerical Definition Document for the Time-explicit ISNaS Solver, ISNaS 88.10.031 (1988)

4. J.L. Thomas, M.D. Salas: Far-Field Boundary Conditions for Transonic Lifting Solutions to the Euler Equations, AIAA Paper 85-0020 (1985)

5. D. Dijkstra: The Solution of the Navier-Stokes Equations near the Trailing Edge of a Flat Plate, Ph. D. Thesis, University of Groningen (1974)

6. A.E.P. Veldman: Boundary Layer Flow past a Finite Flat Plate, Ph. D. Thesis, University of Groningen (1976)

7. P.H. Cook, M.A. McDonald, M.C.P. Firmin: Aerofoil RAE 2822 - Pressure Distributions, and Boundary Layer and Wake Measurements, Agard-AR-138 (1979)

8. T.L. Holst: Viscous Transonic Airfoil Workshop Compendium of Results, AIAA Paper $87-1460$ (1987)

This paper was processed by the authors using the $\mathrm{T}_{\mathrm{E}} \mathrm{X}$ macro package from Springer-Verlag. 